Article

\title{
Operation Scheduling Optimization for Microgrids Considering Coordination of Their Components
}

\author{
Hirotaka Takano ${ }^{1, *}$, Ryota Goto ${ }^{1}$, Thin Zar Soe ${ }^{2}$, Nguyen Duc Tuyen ${ }^{3}\left[\right.$ and Hiroshi Asano ${ }^{1,4}$ \\ 1 Department of Electrical, Electronic and Computer Engineering, Gifu University, Gifu 501-1193, Japan; \\ y4526031@edu.gifu-u.ac.jp (R.G.); hasano@gifu-u.ac.jp (H.A.) \\ 2 PTTEP International Limited (Yangon Branch), Yangon 11041, Myanmar; sweetytina11@gmail.com \\ 3 Hanoi University of Science and Technology, Hanoi 11615, Vietnam; tuyen.nguyenduc@hust.edu.vn \\ 4 Central Research Institute of Electric Power Industry, Yokosuka 240-0196, Japan \\ * Correspondence: takano@gifu-u.ac.jp; Tel.: +81-58-293-2720
}

Received: 5 October 2019; Accepted: 21 October 2019; Published: 24 October 2019

\begin{abstract}
Operation scheduling is one of the most practical optimization problems to efficiently manage the electric power supply and demand in microgrids. Although various microgrid-related techniques have been developed, there has been no established solution to the problem until now. This is because the formulated problem becomes a complicated mixed-integer programming problem having multiple optimization variables. The authors present a framework for this problem and its effective solution to obtain an operation schedule of the microgrid components considering their coordination. In the framework, trading electricity with traditional main power grids is included in the optimization target, and uncertainty originating from variable renewable energy sources is considered. In the solution, the formulated problem is reformulated to reduce the dimensions of its solution space, and, as a result, a combined algorithm of binary particle swarm optimization and quadratic programming is applicable. Through numerical simulations and discussions of their results, the validity of the authors' proposal is verified.
\end{abstract}

Keywords: microgrids; power supply-demand management; unit commitment (UC); economic load dispatch (ELD); binary particle swarm optimization (BPSO); quadratic programming (QP); uncertainty

\section{Introduction}

Microgrids are used in electric power grids to manage a localized group of power sources and loads that can operate in both connecting and disconnecting to the traditional main power grids [1-3]. In association with the growth of renewable energy-based generation systems (REGs), this concept has been attracting attention as one of the most realistic sustainable power grids in terms of efficient use of REGs. Actually, microgrids bring the possibility of grid independence to consumers by improving/keeping the efficiency, reliability, and quality in power supply and demand management in which components of the microgrids can be operated appropriately. Since the early 2000s, extensive research and development has been in progress to figure out efficient solutions for microgrid operations [4]. Furthermore, demonstrative field tests for microgrids have been actively promoted around the world [5-7].

There are two types of microgrid components: controllable and uncontrollable components. The former includes controllable generation systems (CGs), energy storage systems (ESSs), and controllable loads (CLs). A part of the controllable components (e.g., electric vehicles (EVs)), change their attributes depending on the judgement of whether the main grids accept reverse power flow from them or not (accepted: ESS, not accepted: CL). On the other hand, electrical loads in consumer-side and variable REGs (VREGs) (e.g., solar photovoltaic generation systems (PVs) and wind power generation systems 
(WTs)) are in the latter. Since the VREGs, whose outputs strongly depend on the weather condition, take a significant portion of electrical power sources of microgrids, it becomes very difficult to manage the power supply and demand with few adverse effects on the main power grids [1-9]. If microgrid operators cannot maintain the balance of supply and demand, the resulting electricity surplus/shortage must be compensated by trading electricity with an extra payment to the main grids, called imbalance penalty. These are reasons why an operation scheduling method for the microgrids is crucially required.

Focusing on operation of the CGs, the operation scheduling problem in the microgrids is formulated as a mixed-integer programming (MIP) problem that combines optimization problems of the unit commitment (UC) and the economic load dispatch (ELD). This problem is essentially the same as scheduling problems for thermal power generation units [10-16], and, therefore, their solution algorithms are applicable. Branch-and-bound (BB) [17,18] and dynamic programming (DP) [19,20] have been traditionally utilized in thermal power generation scheduling. Meanwhile, intelligent optimization-based techniques have also been involved in solving the problems. Evolutionary programming (EP) [21], genetic algorithms (GAs) [22], simulated annealing (SA) [23,24], tabu search (TS) $[25,26]$, and particle swarm optimization (PSO) $[27,28]$ have been employed in them. Although various traditional and intelligent algorithms have been applied, there has been no established solution until now. Moreover, practical operation scheduling in the microgrids becomes more complicated. This is because installation of the other controllable components brings new optimization variables representing charging/discharging operations of the ESSs and charging operation of the CLs, and their influences cannot be neglected from the microgrid operations. That is, it is necessary to determine the states of all optimization variables simultaneously from the viewpoint of efficient operation of the microgrids $[3,26,27,29-33]$.

Under these circumstances, first, the authors formulate a problem framework to obtain a coordinated operation schedule of the microgrid components. In the problem framework, trading electricity with the main grids is introduced in the optimization target to provide operational alternatives for the microgrid operators. To treat VREG-originated uncertainty, a variation range of the uncertain variables is defined, and the solution candidates (operation schedules) are evaluated using the expected value of operation cost. Next, the formulated problem is reformulated by utilizing its characteristics with the goal to reduce the dimensions of the solution space. As a result of problem reformulation, a combined algorithm of binary particle swarm optimization (BPSO) and quadratic programming (QP) can be applied in the solution. Unlike intelligent algorithms whose solutions much depend on choices of the initial solution and random number sequences [21-28,33], the proposed solution can restrain its dependency and, thus, provide us with more stable solutions. Finally, validity of the problem framework and usefulness of its solution method are verified through numerical simulations and a discussion of their results.

\section{Problem Framework}

\subsection{Overview of Target Problem}

Figure 1 illustrates a typical microgrid model consisting of (1) CGs, (2) ESSs, (3) CLs, (4) electrical loads, and (5) VREGs. As already described, components 1-3 are controllable, and components 4 and 5 are uncontrollable. Detailed definitions of the variables are shown in Section 2.2. The operation scheduling problem in the microgrids is generally formulated to determine a set of start-up/shut-down timing and output shares of component 1 , charging/discharging states of component 2 , and charging states of component 3 in response to the forecasted values of net load on each time interval $[26,27,30,33]$. The net load is calculated by the sum of the forecasted values of electricity consumption of component 4 and output of component 5 .

If the microgrid operators cannot manage the power supply and demand appropriately, the resulting electricity surplus/shortage must be eliminated by trading electricity with the main power grids. In this case, an extra payment is required for compensating the imbalance in the power supply 
and demand as an imbalance penalty. However, electricity trades in the operation scheduling stage have the possibility to reduce complexity in the power supply and demand balancing operations as compared to adjusting operations of the controllable components. In other words, an appropriate operation schedule considering the electricity trade often provides economic benefits for the microgrid operators by improving/keeping the reliability and quality of the power supply. For these reasons, the authors regard electricity trading as an additional controllable component, although components in the main grids cannot be controlled by the microgrid operators.

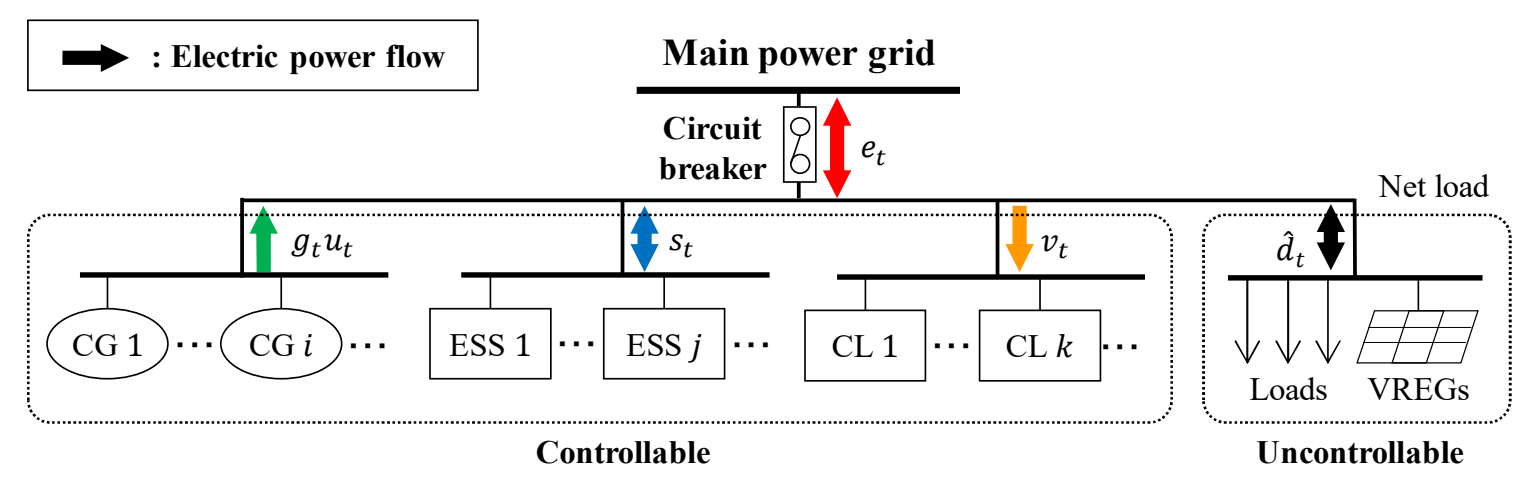

Figure 1. Example of a generalized microgrid model. CG, controllable generation system; ESS, energy storage system; CL, controllable load; VREGs, variable renewable energy-based generation systems.

\subsection{Formulation of Target Problem}

As described in Section 2.1, the operation scheduling problem has five optimization variables defined as

$$
\begin{gathered}
u_{i, t} \in\{0,1\}, \text { for } \forall i, \forall t, \\
g_{i, t} \in\left[G_{i}^{\min }, G_{i}^{\max }\right], \text { for } \forall i, \forall t, \\
s_{j, t} \in\left[S_{j}^{\min }, S_{j}^{\max }\right], \text { for } \forall j, t \in T S_{j}, \\
v_{k, t} \in\left[V_{k}^{\min }, 0\right], \text { for } \forall k, t \in T V_{k}, \\
e_{t} \in \mathbb{R}, \text { for } \forall t,
\end{gathered}
$$

where $t$ is time $(t=1, \cdots, T) ; i$ is the number of CGs $(i=1, \cdots, N G) ; u_{i, t}$ is the ON/OFF state variable of CGs (ON: 1 , OFF: 0 ), which is an element of vectors $u_{t}$ and $u ; g_{i, t}$ is the output of CGs, which is an element of vectors $g_{t}$ and $g_{;} G_{i}^{\max }$ and $G_{i}^{\min }$ are the maximum and the minimum outputs of CGs; $j$ is the number of ESSs $(j=1, \cdots, N S) ; s_{j, t}$ is the output of ESSs and an element of vectors $s_{t}$ and $s ; S_{j}^{\max }$ and $S_{j}^{\min }$ are the maximum and the minimum capable outputs of ESSs $\left(S_{j}^{\min }<0<S_{j}^{\max }\right) ; T S_{j}$ is the set of available time that ESSs are available; $k$ is the number of CLs $(k=1, \cdots, N V) ; v_{k, t}$ is the consumption of CLs and an element of vectors $v_{t}$ and $v ; V_{k}^{\min }$ is the maximum capable consumption of CLs $\left(V_{k}^{\min }<0\right)$; $T V_{k}$ is the set of available time of CLs; and $e_{t}$ is the trading electricity, which is an element of vector $e$.

In general, the operation scheduling problem requires the assumption that forecasted values of the electricity consumption and the VREG outputs are given no matter whether they include uncertainty or not. With a view to treat the uncertainty, the authors express the variable representing the changeable net load with

$$
d_{t} \in\left[d_{t}^{\min }, d_{t}^{\max }\right] \text {, for } \forall t,
$$

where $d_{t}^{\max }$ and $d_{t}^{\min }$ are the maximum and the minimum assumable values of the net load, and they can be set by referring to the historical record.

Fuel costs of the CGs are normally approximated as quadratic functions by means of their outputs and, thus, can be minimized by controlling the CG outputs as steadily as possible. As opposed to the 
CGs, the ESSs consume no fuel in their operations directly, and this makes it challenging to evaluate their operating cost. In this paper, the objective function is represented as

$$
\min _{u, g, s, v, e} \sum_{t=1}^{T} \int_{d_{t}^{\min }}^{d_{t}^{\max }}\left\{C_{t}\left(u_{t}, g_{t}\right)+M_{t} e_{t}+I_{t} e_{t}^{\prime}\right\} f\left(\hat{d}_{t}\right) \mathrm{d} d_{t}
$$

subject to

$$
C_{t}\left(u_{t}, g_{t}\right)=\sum_{i=1}^{N G}\left\{\left(A_{i}+B_{i} g_{i, t}+C_{i} g_{i, t}{ }^{2}\right)+S C_{i}\left(1-u_{i, t-1}\right)\right\} u_{i, t},
$$

where $C\left(u_{t}, g_{t}\right)$ is the operation cost of CGs; $M_{t}$ is the price of electricity trade; $I_{t}$ is the price of the imbalance penalty; $e_{t}^{\prime}$ is the imbalance of electricity; $f\left(\hat{d}_{t}\right)$ is the probability density function for the forecasted net load, $\hat{t}_{t} ; A_{i}, B_{i}$, and $C_{i}$ are the coefficients of fuel cost of CGs; and $S C_{i}$ is the start-up cost of CGs.

Besides, the microgrid must satisfy the below conditions.

- Balance of power supply and demand

$$
d_{t}=\sum_{i=1}^{N G} g_{i, t} u_{i, t}+\sum_{j=1}^{N S} s_{j, t}+\sum_{k=1}^{N V} v_{k, t}+\left(e_{t}+e_{t}^{\prime}\right), \text { for } \forall t
$$

- State duration for CGs

$$
\text { If } 0<u_{i, t}^{o n}<M U T_{i} \text { then } u_{i, t}=1 \text {; If } 0<u_{i, t}^{o f f}<M D T_{i} \text { then } u_{i, t}=0 \text {, for } \forall i, \forall t \text {, }
$$

where $u_{i, t}^{o n}$ and $u_{i, t}^{o f f}$ are the consecutive operating and suspending durations of CGs; and $M U T_{i}$ and $M D T_{i}$ are the minimum operating and suspending durations of CGs.

- Ramp rate for CGs

$$
\Delta G_{i}^{\text {down }} \leq g_{i, t}-g_{i, t-1} \leq \Delta G_{i}^{u p}, \text { for } \forall i, \forall t,
$$

where $\Delta G_{i}^{u p}$ and $\Delta G_{i}^{\text {down }}$ are the ramp-up and the ramp-down rates of CGs.

- Maximum and minimum outputs for CGs

$$
\begin{gathered}
g_{i, t}^{\min } \leq g_{i, t} \leq g_{i, t}^{\max }, \text { for } \forall i, \forall t, \\
\left(g_{i, t}^{\max }=\min \left(G_{i}^{\max }, g_{i, t-1}+\Delta G_{i}^{u p}\right) ; g_{i, t}^{\min }=\max \left(G_{i}^{\min }, g_{i, t-1}+\Delta G_{i}^{\text {down }}\right)\right),
\end{gathered}
$$

where $g_{i, t}^{\max }$ and $g_{i, t}^{\min }$ are the maximum and the minimum outputs for CGs at $t$.

- State for ESSs

$$
\begin{gathered}
Q_{j}^{\min } \leq q_{j, t} \leq Q_{j}^{\max }, \text { for } \forall j, t \in T S_{j}, \\
\left(\text { If } s_{j, t} \leq 0 \text { then } q_{j, t}=q_{j, t-1}-\eta_{j} s_{j, t} ; \text { If } s_{j, t}>0 \text { then } q_{j, t-1}-\frac{1}{\eta_{j}} s_{j, t}\right),
\end{gathered}
$$

where $q_{j, t}$ is the state-of-charge (SOC) level in ESSs; $Q_{j}^{\max }$ and $Q_{j}^{\min }$ are the maximum and the minimum SOC levels of ESSs; and $\eta_{j}$ is the overall efficiency of ESSs.

- Maximum and minimum outputs for ESSs

$$
\begin{gathered}
s_{j, t}^{\min } \leq s_{j, t} \leq s_{j, t}^{\max }, \text { for } \forall j, t \in T S_{j}, \\
\left(s_{j, t}^{\max }=\min \left(S_{j}^{\max }, q_{j, t-1}-Q_{j}^{\min }\right) ; s_{j, t}^{\min }=\max \left(S_{j}^{\min }, q_{j, t-1}-Q_{j}^{\max }\right)\right),
\end{gathered}
$$

where $s_{j, t}^{\max }$ and $s_{j, t}^{\min }$ are the maximum and the minimum outputs for ESSs at $t$. 
- State for CLs

$$
P_{k}^{\min } \leq p_{k, t} \leq P_{k}^{\max }, \text { for } \forall k, t \in T V_{k},\left(p_{k, t}=p_{k, t-1}-\xi_{k} v_{k, t}\right),
$$

where $p_{k, t}$ is the SOC level in CLs; $P_{k}^{\max }$ is the maximum SOC level of CLs; and $\xi_{k}$ is the overall efficiency of CLs.

- Maximum consumption for CLs

$$
v_{k, t}^{\min } \leq v_{k, t} \leq 0, \text { for } \forall k, t \in T V_{k},\left(v_{k, t}^{\min }=\max \left(V_{k}^{\min }, p_{k, t-1}-P_{k}^{\max }\right)\right),
$$

where $v_{k, t}^{\min }$ is the maximum consumption for CLs at $t$.

In summary, the target optimization problem is to determine the operation schedule, $(u, g, s, v, e)$, which minimizes the objective function (7) under constraints (9)-(16). Here, electricity trading is classified into $e_{t}$ and $e_{t}^{\prime}$ for calculating the expected values of the trading cost and the imbalance penalty individually. By using (9), (12), (14), and (16), we can calculate the expected imbalance electricity, $e_{t}^{\prime}$, as

$$
\left\{\begin{array}{c}
\text { If } d_{t}-e_{t}<\sum_{i=1}^{N G} g_{i, t}^{\min } u_{i . t}+\sum_{j=1}^{N S} s_{j, t}^{\min }+\sum_{k=1}^{N V} v_{k, t}^{\min } \text { then } \\
e^{\prime}{ }_{t}=d_{t}-e_{t}-\left(\sum_{i=1}^{N G} g_{i, t}^{\min } u_{i . t}+\sum_{j=1}^{N S} s_{j, t}^{\min }+\sum_{k=1}^{N V} v_{k, t}^{\min }\right) \\
\text { If } d_{t}-e_{t}>\sum_{i=1}^{N G} g_{i, t}^{\max } u_{i . t}+\sum_{j=1}^{N S} s_{j, t}^{\max } \text { then } \\
e_{t}^{\prime}=d_{t}-e_{t}-\left(\sum_{i=1}^{N G} g_{i, t}^{\max } u_{i . t}+\sum_{j=1}^{N S} s_{j, t}^{\max }\right) \\
\text { Else } e_{t}^{\prime}=0
\end{array} .\right.
$$

The reserve margin is automatically secured by (7) and (17) (depending on the expected operational cost in the microgrid).

\section{Solution Method}

\subsection{Overview of Solution Method}

Since every controllable component has a certain influence on the microgrid operations, it is important to determine the states of all optimization variables considering their relationships. However, this problem, as is well known, becomes a complicated MIP problem; therefore, it is hard to solve exactly. Under the circumstances, the application of intelligent optimization algorithms has been regarded as a realistic alternative. Typical algorithms are GA [22], SA [23,24], TS [25,26], and PSO [27,28,33]. In this paper, a BPSO was selected to ease the difficulty of the problems brought by the UC. The ELD problem for each UC candidate, which is created by the paradigm of searching in the BPSO, is solved by a QP solver.

\subsection{Application of Quadratic Programming}

As defined in (1)-(5), the operation scheduling problem has both discrete and continuous optimization variables. The former is $u$, and the latter consists of $g, s, v$ and $e$. With a view to improve compatibility between the target problem and its solution method, the authors redefined the optimization variables as

$$
\begin{gathered}
u_{h, t}^{\prime} \in\{0,1\}, \text { for } \forall h, \forall t, \\
g_{h, t}^{\prime} \in\left[G_{h}^{\min }, G_{h}^{\max }\right], \text { for } \forall h, \forall t,
\end{gathered}
$$

where $h$ is the number of controllable components $(h=1, \cdots, N H ; N H=N G+N S+N V+1) ; u_{h, t}^{\prime}$ is the ON/OFF state variable of controllable components (ON: $1, \mathrm{OFF}: 0$ ), which is an element of vector $u_{h}^{\prime}$ and $u^{\prime} ; g_{h, t}^{\prime}$ is the output of controllable components, which is an element of vector $g_{t}^{\prime}$ and $g^{\prime} ;$ and $G_{h}^{\max }$ and $G_{h}^{\min }$ are the maximum and the minimum outputs of controllable components. 
In (18) and (19), all controllable components are aggregated. The $(N G+1)$-th to the (NG $+N S)$-th components are the ESSs, the $(N G+N S+1)$-th to the $(N H-1)$-th components mean the CLs and the $\mathrm{NH}$-th components represent the trading of electricity. By (7), the sets of coefficients, $\left(A_{h}, B_{h}, C_{h}\right)$, for both the ESS and the CL $(N G<h<N H)$ are $(0,0,0)$, and for the trading electricity $(h=N H)$ they become $(0, M, 0) . S C_{h}(N G<h)$ can be simply set to 0 because there are no start-up costs in each component.

Now, if we fix the states of controllable components on each time, $u_{h, t}^{\prime}$, the target problem can be regarded as a special type of optimization problem that has a quadratic objective function and several variables subject to linear constraints, called the quadratic optimization problem. In this case, QP solvers can be applied; therefore, there is no need to concern the difficulty in determining continuous variables after creating $u^{\prime}$. In other words, the dimensions of the solution space are reduced from $(u, g, s, v, e)$ to $u^{\prime}$, and as a result, we can expect to improve the searching ability of the applied intelligent algorithms.

\subsection{Application of Binary Particle Swarm Optimization}

PSO is a population-based, stochastic computational algorithm [34] that optimizes a problem by iteratively trying to improve a solution candidate for a given measure of quality, called fitness function. An initial set of randomly created solutions, called the initial swarm, propagates in the design of the search space towards the optimal solution over a number of iterations, called moves, based on the large amount of information that is fitted and shared by all members of the swarm. Each particle $x$ has a position, $y_{x}^{m}$, and a velocity, $z_{x}^{m}$, in iteration $m(m=1,2, \ldots, M)$ and flies through the search space to find the best positions and velocities. The inertia weight factor, $\omega$, controls the iteration size. With regards to the proposed problem formulation, the below equations describe the solution update mechanism.

$$
\begin{gathered}
y_{x}^{m}=u^{\prime}, \\
y_{x}^{m+1}=y_{x}^{m}+z_{x}^{m+1}, \\
z_{x}^{m+1}=\omega z_{x}^{m}+\theta_{1} r_{1}\left[y_{x}^{*}-y_{x}^{m}\right]+\theta_{2} r_{2}\left[\left(\min _{x \in X} y_{x}^{*}\right)-y_{x}^{m}\right],
\end{gathered}
$$

where $\theta_{1}$ and $\theta_{2}$ are the cognitive factors that represent the trust for each particle and the swarm; $r_{1}$ and $r_{2}$ are the random numbers in the range, $[0,1] ; y_{x}^{*}$ is the personal best for particle $x$, called pbest; $\min _{x \in X} y_{x}^{*}$ is the best in the swarm, called gbest; and $X$ is the set of all particles.

Although PSO has succeeded in many continuous problems, it still has some difficulties in treating discrete optimization problems [35]. To modify ON/OFF states of the controllable components, $u_{h, t^{\prime}}^{\prime}$ the PSO is expanded as a BPSO, which introduces the following sigmoid function in part of the PSO algorithm.

$$
\text { If } 0.5<\frac{1}{1+e^{-u_{h, t}^{\prime}}} \text { then } u_{h, t}^{\prime}=1 \text {, else } u_{h, t}^{\prime}=0 \text {, for } h<N G, \forall t \text {. }
$$

Here, $u_{h, t}^{\prime}$ for the ESSs and the CLs $(N G<h<N H-1)$ can be set to 1 in the range of available periods of the components (ESSs: $t \in T S_{j}$, CLs: $t \in T V_{k}$ ), while it can be set to 0 in the other periods (ESSs: $t \notin T S_{j}$, CLs: $t \notin T V_{k}$ ). This is because the outputs for the ESSs and the CLs include 0 in their controllable ranges. For the same reason, $u_{h, t}^{\prime}$ for electricity trading $(h=N H)$ can be set to 1 for all time periods. As a result, we can fix $u^{\prime}$, and, therefore, the combined method of BPSO and QP solves the formulated problem approximately. 
With the aim of handling the constraints, the penalty method, which replaces a constrained optimization problem by a series of unconstrained problems, is applied during the iterative process. The fitness function is represented as

$$
F=\sum_{t=1}^{T}\left\{\int_{d_{t}^{\min }}^{d_{t}^{\max }}\left\{C_{t}\left(u_{t}, g_{t}\right)+M_{t} e_{t}+I_{t} e_{t}^{\prime}+V I O_{t}\right\} f\left(d_{t}\right) \mathrm{d} d_{t}\right\},
$$

where $\mathrm{VIO}_{t}$ is the weighted sum of violation of the constraints excluding (9) $\left(\mathrm{VIO}_{t} \gg \mathrm{C}_{t}\left(u_{t}, g_{t}\right)+\right.$ $\left.M_{t} e_{t}+I_{t} e_{t}^{\prime}\right)$.

\section{Numerical Simulations}

\subsection{Numerical Conditions}

Numerical simulations were carried out on the microgrid model, which was already illustrated in Figure 1, to verify the validity of the authors' proposal. Figure 2 shows profiles of the forecasted net load and the price in electricity trade. As shown in Figure 2, the aggregated PV on sunny day was referred to as the aggregated VREG. The imbalance penalty was set to a large value to avoid the electricity surplus/shortage in the operation stage. Specifications of the controllable components in the microgrid model are summarized in Tables 1 and 2. Here, the ESSs and the CLs were aggregated into one large-scale component, respectively, to simplify discussions. These were made by referring to $[26,27,33,36]$.

Time interval, $\Delta t$, was set to $1 \mathrm{~h}$, and daily operation schedules $(t=1,2, \ldots, 24)$ were determined. However, the period of optimization target was set from 8:00 $\mathrm{AM}(t=1)$ to 7:00 $\mathrm{AM}(t=24)$ considering operation of the time-constrained components in the microgrid model. The available duration of the CLs was set from 9:00 PM to 7:00 AM. The initial SOC level of the aggregated ESS was set to 50\% of its capacity, and the level had to be returned to the original state until the end of the scheduling period. On the other hand, the initial SOC level of the aggregated CL was set to $50 \%$, and it had to be $100 \%$ at the end of the scheduling period.

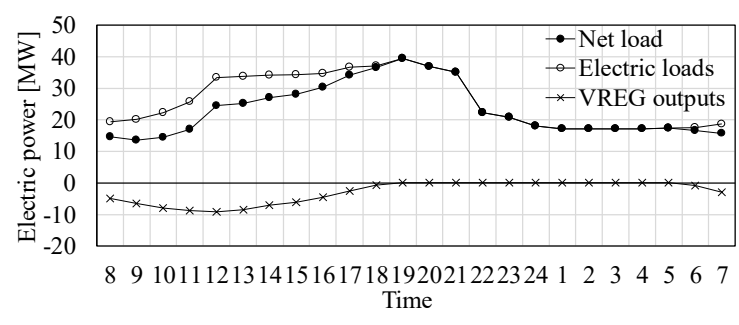

(a)

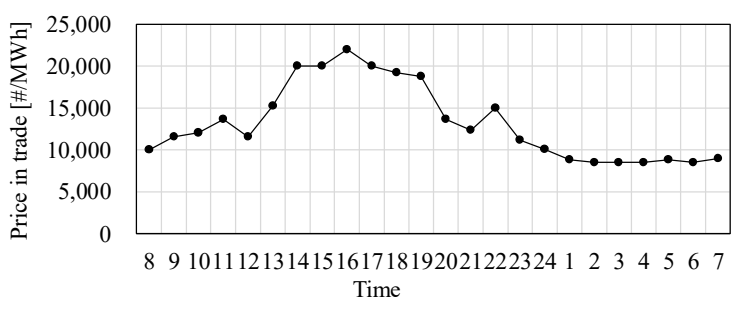

(b)

Figure 2. (a) Forecasted net load; (b) electricity price.

Table 1. Specification of CGs ("\#" means "any currency unit is applicable").

\begin{tabular}{ccccccc}
\hline $\boldsymbol{i}$ & $\boldsymbol{A}_{\boldsymbol{i}}$ (\#) & $\boldsymbol{B}_{\boldsymbol{i}}(\# / \mathbf{M W})$ & $\boldsymbol{C}_{\boldsymbol{i}}\left(\# / \mathbf{M W}^{2}\right)$ & $S C_{i}(\#)$ & $G_{i}^{\text {max }}(\mathbf{M W})$ & $G_{i}^{\text {min }}(\mathbf{M W})$ \\
\hline 1 & $12,000.0$ & 3800.0 & 1.2 & 3000.0 & 20.0 & 4.0 \\
2 & 7800.0 & 3100.0 & 1.8 & 1000.0 & 16.0 & 3.2 \\
3 & 2400.0 & 2500.0 & 2.8 & 500.0 & 12.0 & 2.4 \\
\hline
\end{tabular}

Table 2. Specifications of aggregated ESS and aggregated CL.

\begin{tabular}{ccccccc}
\hline$S^{\max }(\mathrm{MW})$ & $S^{\min }(\mathrm{MW})$ & $Q^{\max }(\mathbf{M W h})$ & $Q^{\min }(\mathrm{MWh})$ & $V^{\max }(\mathrm{MW})$ & $P^{\max }(\mathbf{M W h})$ & $P^{\min }(\mathrm{MWh})$ \\
\hline 1.8 & -1.8 & 10.4 & 2.6 & -1.5 & 9.6 & 2.4 \\
\hline
\end{tabular}


Under these conditions, the authors determined the following three operation schedules by the proposed solution method:

Case 1 Operation schedule without the reserve margin.

Case 2 Operation schedule considering the conventional reserve margin that compensates deviation within $5 \%$ of the net load.

Case 3 Operation schedule based on the proposed framework.

In accordance with the results of preliminary trials and errors, the parameters for BPSO were set as follows: $|X|=40, M=300, \omega=0.9, \theta_{1}=2.0$, and $\theta_{2}=2.0$.

\subsection{Results and Discussion}

Figures $3-5$ and Table 3 summarize the numerical results. As shown in Figures $3-5$, the balance of power supply and demand for the forecasted net load was maintained by the sum of output shares of the CGs, the aggregated ESS and the aggregated CL, and the trading electricity in all cases. In Cases 1 and 2, the numerical simulations succeeded within a few minutes. However, Case 3 required a few hours to obtain the optimal operation schedule since evaluation of the UC candidates (calculation by the QP solver) became complicated. Therefore, improvement in the computational speed remains an important issue in the authors' proposal, although the process can be accomplished from the perspective of practical use.

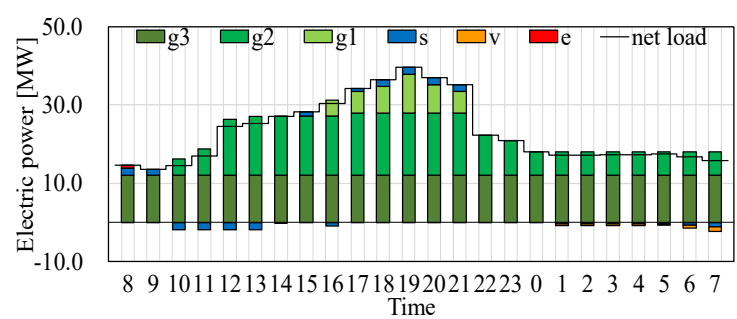

(a)

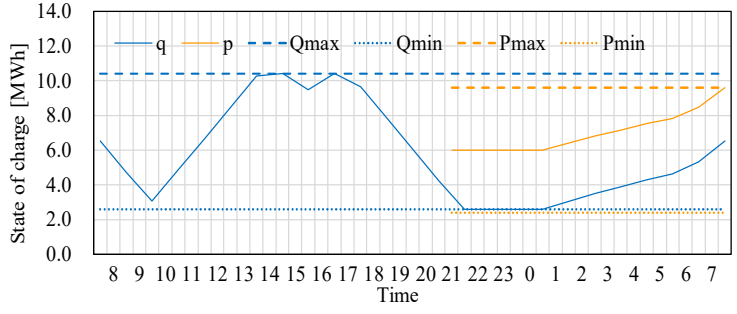

(b)

Figure 3. (a) Operation schedule for forecasted net load in Case 1; (b) state-of-charge (SOC) level in Case 1.

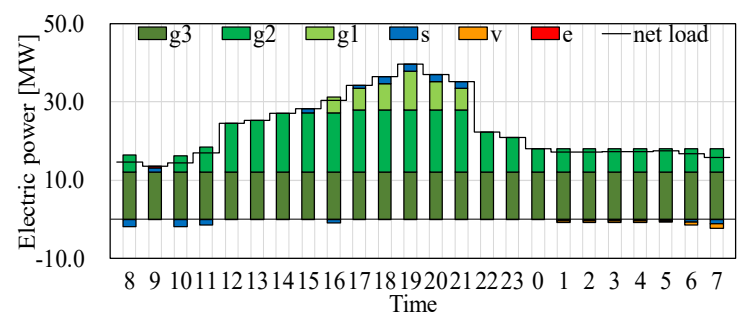

(a)

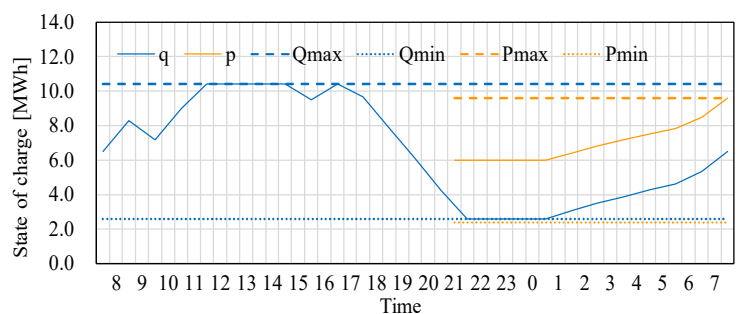

(b)

Figure 4. (a) Operation schedule for forecasted net load in Case 2; (b) SOC level in Case 2.

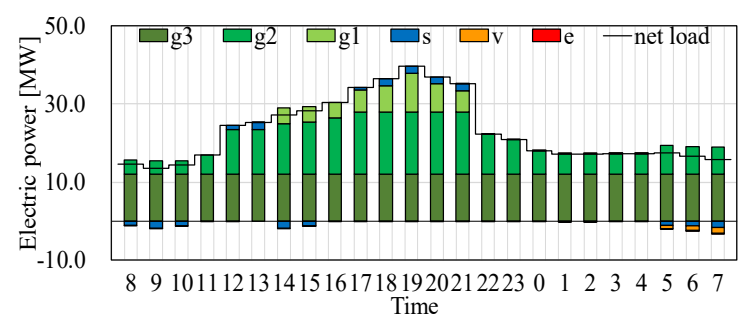

(a)

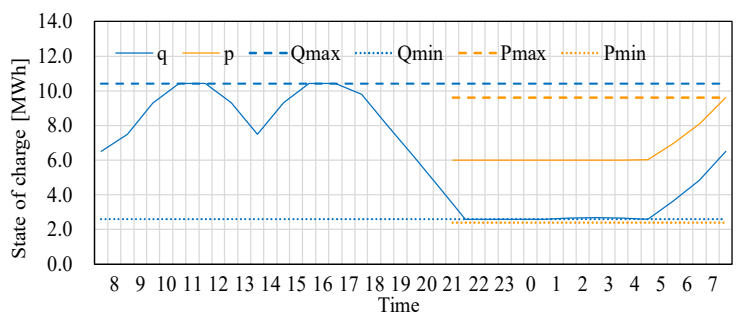

(b)

Figure 5. (a) Operation schedule for forecasted net load in Case 3; (b) SOC level in Case 3. 
Table 3. Comparison of numerical results.

\begin{tabular}{ccc}
\hline Case & Cost for Forecasted Net Load (\#) & Expected Cost (\#) \\
\hline 1 & $1,921,509.5$ & $2,101,022.6$ \\
2 & $1,929,108.8$ & $2,051,418.4$ \\
3 & $1,960,789.9$ & $1,960,832.3$ \\
\hline
\end{tabular}

In Table 3, the operation schedule of Case 1 was the best result for the forecasted net load. It means that the operational cost increased in the other cases for securing the reserve margin against the uncertainty in the actual operation. Besides, it was found that the expected values of the operation costs became worse than the operation costs for the forecasted net load in each case. However, in Case 3 , the increase in the objective function was not so large as compared to that in Case 2 in spite of the potential risk that was reflected in both cases. As a result, the expected cost in Case 3 was the smallest as opposed to the operation cost for the forecasted net load. From these results, we can conclude that the authors' proposal functioned well.

\section{Conclusions}

This paper presented a problem framework and its effective solution to obtain an operation schedule to coordinate microgrid components considering. In the problem framework, trading of electricity was included in the optimization target, and VREG-originated uncertainty was considered. Moreover, the formulated problem was reformulated to reduce the dimensions of its solution space; therefore, the BPSO-QP was applicable in the solution method. Through numerical simulations and a discussion of their results, we can conclude that the proposed problem framework and its solution method functioned appropriately.

In future works, the authors will improve the calculation time of BPSO-QP. In addition, the authors will propose more effective solutions with respect to the characteristics of the target optimization problem.

Author Contributions: Conceptualization, H.T., R.G., T.Z.S. and H.A.; methodology, H.T., R.G. and T.Z.S.; validation, H.T., R.G. and N.D.T.; writing-original draft preparation, H.T. and N.D.T.; writing-review and editing, H.T. and T.Z.S.; supervision, H.T. and H.A.

Funding: This research was funded by Japan Society for the Promotion of Science (JSPS), grant numbers 16K06215 and 19K04325.

Acknowledgments: The authors would like to acknowledge Gifu Renewable Energy System Research Center of Gifu University.

Conflicts of Interest: The authors declare no conflicts of interest.

\section{References}

1. Office of Electricity Delivery and Energy Reliability. DOE Microgrid Workshop Report. Available online: https://www.energy.gov/sites/prod/files/2012\%20Microgrid\%20Workshop\%20Report\%2009102012.pdf (accessed on 31 May 2019).

2. Ton, D.T.; Smith, M.A. The U.S. Department of Energy's Microgrid Initiative. Electr. J. 2012, $25,84-94$. [CrossRef]

3. Bevrani, H.; Francois, B.; Ise, T. Microgrid Dynamics and Control; Wiley: Hoboken, NJ, USA, 2017.

4. Hatziargyriou, N.; Asano, H.; Iravani, R.; Marnay, C. Microgrids for Distributed Generation. IEEE Power Energy Mag. 2007, 133, 144-149.

5. Investigating R\&D Committee on advanced power system. Current Status of Advanced Power Systems including Microgrid and Smartgrid. IEEJ Tech. Rep. 2011, 1229. Available online: https://www.bookpark.ne. jp/cm/ieej/detail.asp?content_id=IEEJ-GH1229-PRT (accessed on 24 October 2019).

6. New Energy and Industrial Technology Development Organization. Case Studies of Smart Community Demonstration Project. Available online: http://www.nedo.go.jp/english/reports_20130222.html (accessed on 31 May 2019). 
7. Choi, Y.J.; Choi, I.S.; Lee, H.J.; Chae, W.K. Operational Experience of Microgrids. In Smart Grid Handbook, 3 Volume Set; Liu, C.C., McAuthur, S., Lee, S.J., Eds.; Wiley: Hoboken, NJ, USA, 2016; Volume 3, pp. 1213-1234.

8. Chowdhury, D.; Hassan, A.S.M.K.; Khan, M.Z.R. Scalable DC Microgrid Architecture with Phase Shifted Full Bridge Converter Based Power Management Unit. In Proceedings of the 10th International Conference on Electrical and Computer Engineering, Dhaka, Bangladesh, 20-22 December 2018; pp. 22-25.

9. Hassan, A.S.M.K.; Chowdhury, D.; Khan, M.Z.R. Scalable DC Microgrid Architecture with a One-Way Communication Based Control Interface. In Proceedings of the 10th International Conference on Electrical and Computer Engineering, Dhaka, Bangladesh, 20-22 December 2018; pp. 265-268.

10. Kerr, R.H.; Scheidt, J.L.; Fontana, A.J.; Wiley, J.K. Unit Commitment. IEEE Trans. Power A Syst. 1996, PAS-85, 417-421. [CrossRef]

11. Sen, S.; Kothari, D.P. Optimal Thermal Generating Unit Commitment: A Review. Int. J. Electr. Power Energy Syst. 1998, 20, 443-451. [CrossRef]

12. Hobbs, B.F.; Rothkopf, M.H.; O'Neill, R.P.; Chao, H.P. The Next Generation of Electric Power Unit Commitment Models; Springer: New York City, NY, USA, 2001.

13. Padhy, N.P. Unit Commitment-A Bibliographical Survey. IEEE Trans. Power Syst. 2004, 19, 1196-1205. [CrossRef]

14. Bhardwaj, A.; Tung, N.S.; Kamboj, V. Unit Commitment in Power System: A Review. Int. J. Power Eng. 2012, 6, 51-57. [CrossRef]

15. Saravanan, B.; Das, S.; Sikri, S.; Kothari, D.P. A Solution to the Unit Commitment Problem-A Review. Front. Energy 2013, 7, 223-236. [CrossRef]

16. Zheng, Q.P.; Wang, J.; Liu, A.L. Stochastic Optimization for Unit Commitment-A Review. IEEE Trans. Power Syst. 2015, 30, 1913-1924. [CrossRef]

17. Cohen, A.I.; Yoshimura, M. A branch-and-Bound Algorithm for Unit Commitment. IEEE Trans. Power A Syst. 1983, PAS-102, 444-451. [CrossRef]

18. Chen, C.L.; Wang, S.C. Branch-and-Bound Scheduling for Thermal Generating Units. IEEE Trans. Energy Convers. 1993, 8, 184-189. [CrossRef]

19. Snyder, W.L.; Powell, H.D.; Raiburn, J.C. Dynamic Programming Approach to Unit Commitment. IEEE Trans. Power Syst. 1987, 2, 339-348. [CrossRef]

20. Ouyang, Z.; Shahidehpour, S.M. An Intelligent Dynamic Programming for Unit Commitment Application. IEEE Trans. Power Syst. 1991, 6, 1203-1209. [CrossRef]

21. Juste, K.A.; Kita, H.; Tanaka, E.; Hasegawa, J. An Evolutionary Programming Solution to the Unit Commitment Problem. IEEE Trans. Power Syst. 1990, 14, 1452-1459. [CrossRef]

22. Kazarlis, S.A.; Bakirtzis, A.G.; Petridis, V. A Genetic Algorithm Solution to the Unit Commitment Problem. IEEE Trans. Power Syst. 1996, 11, 83-92. [CrossRef]

23. Mantawy, A.H.; Abdel-Magid, Y.L.; Selim, S.Z. A Simulated Annealing Algorithm for Unit Commitment. IEEE Trans. Power Syst. 1998, 13, 197-204. [CrossRef]

24. Simopoulos, D.N.; Kavatza, S.D.; Vournas, C.D. Unit Commitment by an Enhanced Simulated Annealing Algorithms. IEEE Trans. Power Syst. 2006, 21, 68-76. [CrossRef]

25. Rajan, C.C.A.; Mohan, M.R. An Evolutionary programming-based Tabu Search Method for Solving the Unit Commitment Problem. IEEE Trans. Power Syst. 2004, 19, 577-585. [CrossRef]

26. Takano, H.; Zhang, P.; Murata, J.; Hashiguchim, T.; Goda, T.; Iizaka, T.; Nakanishi, Y. A determination method for the optimal operation of controllable generators in micro grids that copes with unstable outputs of renewable energy generation. Electr. Eng. Jpn. 2015, 190, 56-65. [CrossRef]

27. Hayashi, Y.; Miyamoto, H.; Matsuki, J.; Iizuka, T.; Azuma, H. Online Optimization Method for Operation of Generators in Micro Grid. IEEJ Trans. PE 2008, 128, 388-396. (In Japanese) [CrossRef]

28. Jeong, Y.W.; Park, J.B. A New Quantum-Inspired Binary PSO: Application to Unit Commitment Problem for Power Systems. IEEE Trans. Power Syst. 2010, 25, 1486-1495. [CrossRef]

29. Lu, B.; Shahidehpour, M. Short-Term Scheduling of Battery in a Grid-Connected PV/Battery System. IEEE Trans. Power Syst. 2005, 20, 1053-1061. [CrossRef]

30. Palma-Behnke, R.; Benavides, C.; Lanas, F.; Severino, B.; Reyes, L.; Llanos, J.; Saez, D. A Microgrid Energy Management System Based on the Rolling Horizon Strategy. IEEE Trans. Smart Grid. 2013, 4, 996-1006. [CrossRef] 
31. Li, N.; Uckun, C.; Constantinescu, E.M.; Birge, J.R.; Hedman, K.W.; Botterud, A. Flexible Operation of Batteries in Power System Scheduling with Renewable Energy. IEEE Trans. Sustain. Energy 2016, 7, 685-696. [CrossRef]

32. Hammati, R.; Saboori, H. Short-Term Bulk Energy Storage Scheduling for Load Leveling in Unit Commitment: Modeling, Optimization, and Sensitivity Analysis. J. Adv. Res. 2016, 7, 360-372. [CrossRef] [PubMed]

33. Soe, T.Z.; Takano, H.; Shiomi, R.; Taoka, H. Determination method for optimal cooperative operation plan of microgrids by providing alternatives for microgrid operators. J. Int. Counc. Electr. Eng. 2018, 8, 103-110. [CrossRef]

34. Clerc, M. Particle Swarm Optimization; Wiley: Hoboken, NJ, USA, 2006.

35. Lee, S.; Soak, S.; Oh, S.; Pedryczm, W.; Jeon, M. Modified binary particle swarm optimization. Prog. Nat. Sci. 2008, 18, 1161-1166. [CrossRef]

36. Takano, H.; Nagaki, Y.; Murata, J.; Iizaka, T.; Ishibashi, T.; Katsuno, T. A study on supply and demand planning for Power Producer-Suppliers utilizing output of megawatt solar plants. J. Int. Counc. Electr. Eng. 2016, 6, 102-109. [CrossRef]

(C) 2019 by the authors. Licensee MDPI, Basel, Switzerland. This article is an open access article distributed under the terms and conditions of the Creative Commons Attribution (CC BY) license (http://creativecommons.org/licenses/by/4.0/). 